\title{
Orbiter High Resolution Camera onboard Chandrayaan-2 Orbiter
}

\author{
Arup Roy Chowdhury*, Manish Saxena, Ankush Kumar, S. R. Joshi, \\ Amitabh, Aditya Dagar, Manish Mittal, Shweta Kirkire, Jalshri Desai, \\ Dhrupesh Shah, J. C. Karelia, Anand Kumar, Kailash Jha, Prasanta Das, \\ H. V. Bhagat, Jitendra Sharma, D. N. Ghonia, Meghal Desai, Gaurav Bansal \\ and Ashutosh Gupta
}

Space Applications Centre, Indian Space Research Organisation, Ahmedabad 380 015, India

Orbiter High Resolution Camera (OHRC) onboard Chandrayaan-2 Orbiter-craft, is a very high spatial resolution camera operating in visible panchromatic band. OHRC's primary goal is to image the landingsite region prior to landing for characterization and finding hazard-free zones. Post landing operation of the OHRC will be for scientific studies of small-scale features on the lunar surface. OHRC makes use of the time delay integration detector to have good signal-tonoise ratio under low illumination condition and less integration time due to very high spatial resolution. Ground sampling distance (GSD) and swath of OHRC (in nadir view) are $0.25 \mathrm{~m}$ and $3 \mathrm{~km}$ respectively, from $100 \mathrm{~km}$ altitude. GSD is better than $0.32 \mathrm{~m}$ in oblique view (25 ${ }^{\circ}$ pitch angle) during landing site imaging from $100 \mathrm{~km}$ altitude in two stereo views in consecutive orbits. This article includes the details of the configuration, sub-systems, imaging modes, and optical, spectral and radiometric characterization performance.

Keywords: Ground sampling distance, orbiter high resolution camera, relative spectral response, square wave response, time delay integration.

CHANDRAYAAN-2, India's second lunar mission, has orbiter-craft, lander-craft and rover as a composite module. The orbiter-craft will have a circular polar orbit at an altitude of $100 \mathrm{~km}$. Orbiter High Resolution Camera (OHRC) is one of the important instruments onboard the orbiter-craft. OHRC has $0.25 \mathrm{~m}$ ground sampling distance (GSD) and $3 \mathrm{~km}$ swath at nadir from $100 \mathrm{~km}$. OHRC makes use of the Ritchey-Chretien (RC)-telescope with field correcting optics (FCO) and $12 \mathrm{~K}$ by 256 time delay integration (TDI) detector. Stereo imaging is through the spacecraft orientation and manoeuvring to have desired view angles. Landing site is in highland region with typical reflectance of about $8 \%$ in the visible range. OHRC

*For correspondence. (e-mail: arc@sac.isro.gov.in) can image lunar surface under low illumination condition (Sun elevation angle about $5-6^{\circ}$ ). This is an important requirement to have early landing in the lunar day-cycle and maximize the number of days available for Landercraft and Rover instrument operation.

\section{Objectives}

There are broadly two type of objectives of OHRC namely 'mission objectives' and 'science objectives'. Mission objective is prime for the safe landing of the Chandrayaan-2 lander-craft by making use of the OHRC images and derived datasets like crater catalogue and hazard map (for boulders, craters, slopes and shadows).

\section{Mission objectives}

OHRC's mission objective is to capture images of the Moon surface with high spatial resolution to characterize the landing site (prior to landing) with a GSD better than $0.32 \mathrm{~m}$ during two oblique views $(0.25 \mathrm{~m}$ at nadir $)$ from $100 \mathrm{~km}$ altitude. Nominal OHRC imaging region of landing site region is about $3 \mathrm{~km} \times 12 \mathrm{~km}$ area in two orbits with different view angles (with spacecraft maneuvering) to generate digital elevation model (DEM) information. Major hazards for lander-craft are slopes, boulders, craters and shadows for which OHRC data is important. Slope from DEM and shadows from brightness images are required to generate the hazard map and safe-grid location.

OHRC observation is important to update the landing grid and the landing path (i.e. trajectory). OHRC data processing is at ground to generate the required data sets for lander-craft (e.g. updated crater catalogue, hazard map or safe-grid map), required to assist other sensors during various phases of the landing or descent trajectory (e.g. land-mark identification, final safe-grid identification during hovering). Locating and monitoring of the Lander-craft and Rover by OHRC is the post-landing OHRC imaging activity. 
Table 1. Parameters of Orbiter High Resolution Camera

\begin{tabular}{ll}
\hline Parameter & \multicolumn{1}{c}{ Values } \\
\hline Orbit altitude $(\mathrm{km})$ & 100 \\
GSD $(\mathrm{m})$ at nadir & 0.25 \\
Swath $(\mathrm{km})$ at nadir & 3 \\
Spectral range $(\mathrm{nm})$ & $450-800$ \\
Telescope diameter $(\mathrm{mm})$ & 300 \\
Detector & $12 \mathrm{~K}$ by 256 TDI \\
Quantization (bits) & 10 (electronics) 8 (transmission) \\
Reference illumination condition & $8 \%$ Albedo, 5-6 ${ }^{\circ}$ Sun elevation \\
Reference radiance $\left(\mathrm{mW} / \mathrm{cm}^{2} / \mathrm{sr} / \mu \mathrm{m}\right)$ & 0.5 \\
Saturation radiance $\left(\mathrm{mW} / \mathrm{cm}^{2} / \mathrm{sr} / \mu \mathrm{m}\right)$ & 0.8 with 256 TDI \\
Signal-to-noise ratio $(\mathrm{SNR}) @$ reference radiance & 100 with 256 TDI (140 at saturation) \\
Stereo views & Fore and Aft in two consecutive orbits \\
& by spacecraft and maneuvering \\
\hline
\end{tabular}

Table 2. Optical system parameters

\begin{tabular}{ll}
\hline Parameter & \multicolumn{1}{c}{ Value } \\
\hline Optical configuration & $\begin{array}{c}\text { Ritchey-Chretien telescope with field } \\
\text { correcting optics (RC + FCO) }\end{array}$ \\
Spectral range (nm) & $450-800$ \\
Primary mirror diameter, $D(\mathrm{~mm})$ & 300 \\
Effective focal length (EFL; mm) & 2046 \\
Field-of-view (FOV, deg) & \pm 0.86 \\
MTF (\%)@96 lp/mm (Nyquist) & 24 \\
\hline
\end{tabular}

Table 3. Detector parameters

\begin{tabular}{ll}
\hline Parameter & \multicolumn{1}{c}{ Value } \\
\hline Detector type & TDI-CCD \\
Pixel size $(\mu \mathrm{m})$ & $5.2 \times 5.2$ \\
Spectral range $(\mathrm{nm})$ & $450-800$ \\
TDI stages & $256,192,128,64$ (selectable) \\
Full well capacity $\left(\mathrm{ke}^{-}\right)$ & 26.6 \\
Read noise $\left(\mathrm{e}^{-}\right)$ & 40 \\
\hline
\end{tabular}

\section{Science objectives}

OHRC's science objectives are supported by its very high spatial resolution imaging capability. Also low illumination imaging capability adds values to the observations in the near polar regions. It can image small-scale features in fine detail. The first objective of OHRC, after mission objective, i.e. the landing-site imaging, is to image the anthropogenic sites on lunar surface. The other scientific objectives are: (i) to study the recent volcanism and other small-scale volcanic sites; (ii) to study the central peaks of young impact craters, and (iii) to understand tectonic (seismic) processes (through boulder population determination, boulder rolling trails, seismicity related features, etc.); impact cratering (small craters, ejecta material, melt pools, etc.) and mass wasting (rock falls, debris flow, etc.).

\section{System configuration}

OHRC has $0.25 \mathrm{~m}$ GSD and $3 \mathrm{~km}$ swath from $100 \mathrm{~km}$ altitude. It operates in visible $(450-800 \mathrm{~nm})$ panchromatic band. It makes use of the RC-telescope with FCO to collect the signal and image onto a 256 stage TDI-detector with 12,000 pixels. RC-telescope is a high focal length and compact optical system. TDI detector is required to collect sufficient signal under low illumination and less integration time condition. TDI stages and integration time can be selected through telecommand (TC). Table 1 gives the parameters of the OHRC.

\section{Optical system}

To collect adequate signal for meeting the SNR requirement, optical aperture diameter of $300 \mathrm{~mm}$ for primary mirror is selected. For this aperture, reflective system has less weight and is realizable. Considering pixel size of the detector, effective focal length (EFL) requirement is $\sim 2 \mathrm{~m}$ to have $0.25 \mathrm{~m}$ GSD from $100 \mathrm{~km}$. To have $3 \mathrm{~km}$ swath, field-of-view requirement is $\pm 0.86^{\circ}$. Thus, RitcheyChretien telescope with field correcting optics (i.e. $\mathrm{RC}+\mathrm{FCO}$ ) configuration is selected to meet the large focal length and field-of-view requirement. Figure 1 shows the optical configuration of OHRC. Table 2 provides the optical system parameters. 
Table 4. Camera electronics parameters

\begin{tabular}{lc}
\hline Parameter & \\
\hline Quantization (bit) & 10 (electronics) \\
& 8 (transmission) by bit selection \\
Integration times $(\mu \mathrm{s})$ & 7 values (selectable by telecommand (TC)) 213.84, 209.58, 205.32, 181.74, \\
& $178.14,174.87$ (for landing site imaging catering to $\pm 2 \mathrm{~km}$ altitude variation, \\
& fore and aft view) and $162.10 \mu \mathrm{s}$ (for Nadir view imaging) \\
TDI stages & $256,192,128,64$ (selectable by TC) 256 (for landing site) 64 (post-landing) \\
Data volume (per view) & 4.6 Gb for one $3 \mathrm{~km} \times 12 \mathrm{~km}$ strip (for 8 bits) \\
Raw power $(\mathrm{W})$ & $\sim 36$ \\
\hline
\end{tabular}

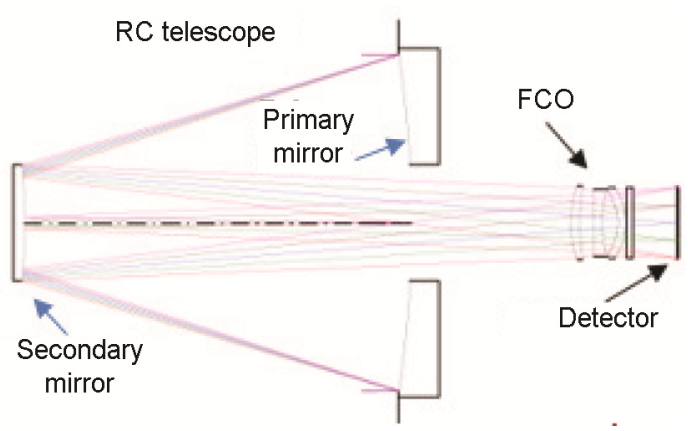

Figure 1. Optical configuration.

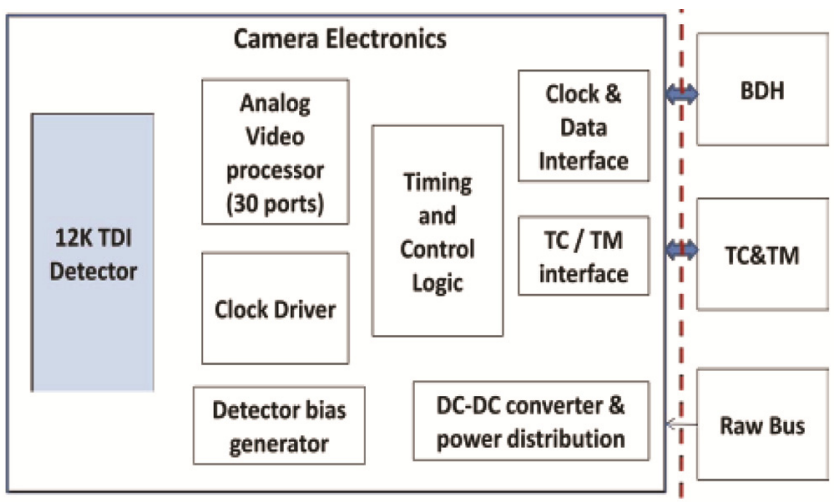

Figure 2. Camera electronics.

A trade-off study was carried out for selecting the spectral range of the complete system considering the SNR and modulation transfer function (MTF) performance. Spectral range of $450-800 \mathrm{~nm}$ was found to be optimum. Extending spectral range (specifically towards higher wavelengths) was found to have negative impact on the MTF performance without much gain in the signal collection.

\section{Detector}

To meet the mission requirements in terms of GSD, swath and SNR; a commercial of the shelf (COTS) TDI-CCD detector was considered. This detector was qualified in-house for flight use. It has pixel size of $5.2 \mu \mathrm{m}$, format of 12,000 pixels and up to 256 TDI stages with four selectable values. System level measurement shows that readout noise is about $40 \mathrm{e}^{-}$and full-well capacity is $26.6 \mathrm{ke}^{-}$. Table 3 provides the detector parameters.

\section{Camera electronics}

To operate detector and acquire data, camera electronics (CE) generates required clocks, biases and control signals. CE consists of detector proximity electronics (DPE), front end camera electronics (FECE), logic and control electronics (LCE) and power supply electronics (PSE). CE block diagram is shown in Figure 2.

DPE provides interface of clocks, biases and output video data from detector to FECE.

FECE provides low noise DC biases, clocks (of suitable voltage level) for detector operation, acquiring video data and data preprocessing. FECE also do analog to digital conversion, serialization and data interface to the spacecraft's baseband data handling (BDH) system.

LCE generates the clocks for FECE and interfaces with BDH for clocks and data. LCE also interfaces with the spacecraft's telecommand and telemetry (TC\&TM) system.

PSE draws power from the spacecraft and caters to the power requirements of DPE, FECE and LCE. Table 4 provides the CE parameters.

\section{Mechanical and thermal system}

To house the optical module, detector head assembly (DHA) and CE, a mechanical system is required which includes structure, packages and other components of electro-optical module (EOM) like baffles, fixtures, couplers, alignment cube, etc. (Figure 3). Thermal control in the instrument ensures that all sensitive elements remain within the specified temperature limits, considering about $300^{\circ} \mathrm{C}$ temperature difference to be viewed by the instrument over the Moon's surface. The thermal control is achieved by passive thermal control techniques augmented with auto/commandable heaters.

CURRENT SCIENCE, VOL. 118, NO. 4, 25 FEBRUARY 2020 
OHRC is mounted on the negative pitch (-P) panel of the orbiter-craft. Table 5 shows the physical parameters of OHRC. Figure 3 shows the CAD model for EOM of OHRC. It shows various components like mirrors, cylinder, FCO, DHA, baffles and alignment cube.

\section{Imaging modes}

\section{Earth bound phase imaging mode for Earth and deep space observation}

This mode is planned to observe Earth during the Earth bound orbital phase for functional testing. Suitable spacecraft maneuvering and scan rate is required for this operation. Deep space observation for dark data is required for dark noise performance study.

\section{Lunar bound phase test-imaging mode for lunar observation}

This mode is for 'readiness for landing site imaging'. It is required to observe Lunar surface few orbits before the nominal imaging sessions for the landing site. This is required for functional test and initial processing verification and readiness at ground for actual landing site observation and processing.

\section{Pre-landing nominal-imaging mode for lunar observation}

This mode is to meet the 'mission objective'. This is the nominal observation of the actual landing site for its characterization with respect to hazards and safe zones. Landing site observation with two view angles in two consecutive orbits is configured by spacecraft maneuvering (Figure 4). Two stereo views are $+5^{\circ}$ and $-25^{\circ}$ about the pitch axis. Also roll tilt $23^{\circ}$ (orbit\# -4) and $17.6^{\circ}$ (orbit\# -3) respectively is required to ensure proper side view of the landing site few orbits prior to landing orbit. Time period of the satellite is about $2 \mathrm{~h}$. Time taken for imaging the landing site (i.e. $3 \mathrm{~km} \times 12 \mathrm{~km}$ strip) is about $8 \mathrm{sec}$ per stereo view, every orbit.

\section{Post-landing imaging mode for lunar observation}

This mode is to meet the 'science objective'. After landing of lander, OHRC is to be used for nominal high resolution imaging of the lunar surface. It can also be used for imaging lander and rover after landing. Nominal integration time for Nadir view imaging is $162.1 \mu$ s for nominal spacecraft altitude of $100 \mathrm{~km}$. Due to higher illumination during post-landing imaging scenario, lower TDI stages (e.g. 64) can be selected among four options (64, 128, 192 and 256 TDI stages) by TC.

\section{Post-landing imaging mode for Earth and deep} space observation

This mode is planned for observing Earth during the Moon bound orbital phase after landing operation for scientific interest and outreach. Suitable spacecraft maneuvering and scan rate is required for this operation. Deep space observation for dark data is required for dark noise performance study.

\section{Payload characterization}

Payload characterization results are discussed in this section. Figure 5 shows the realized OHRC payload.

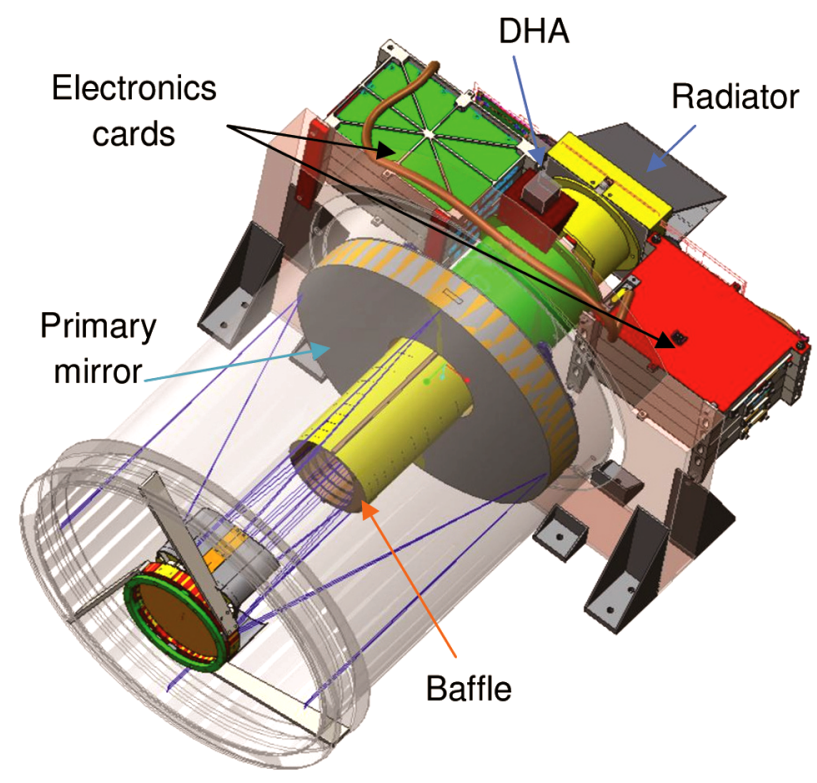

Figure 3. CAD model of electro-optical module of OHRC.

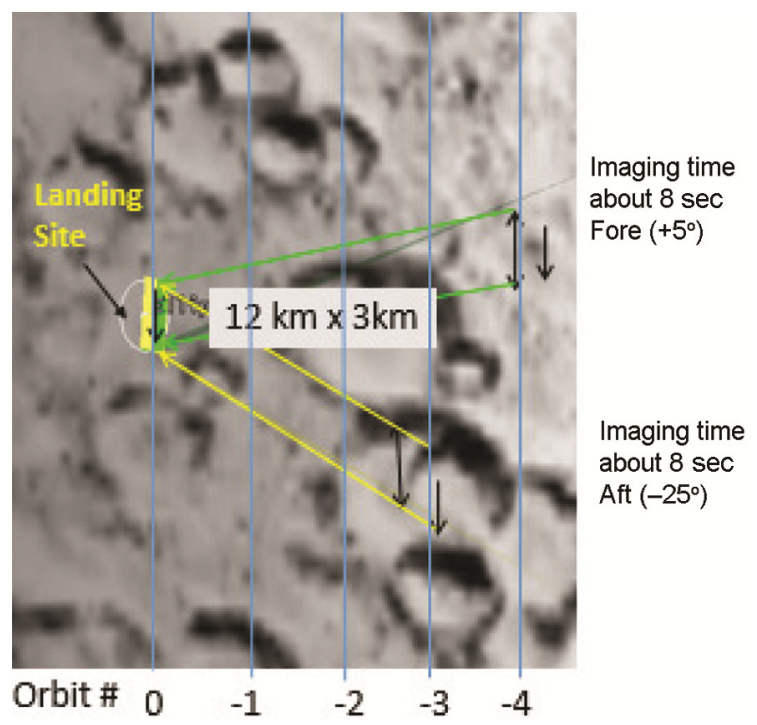

Figure 4. Typical schematic of tilted view observation with tilt about pitch axis. 


\section{RESEARCH ARTICLES}

\section{Optical characterization - SWR measurement}

Square wave response (SWR) is the important parameter of the image quality of a camera. SWR indicates the sharpness or contrast of the features in the image. SWR is measured for the OHRC's central as well extreme fields at Nyquist (Nq, i.e. $97 \mathrm{lp} / \mathrm{mm}$ ) as well as $\mathrm{Nq} / 2$ (48 lp/mm) spatial frequency (Table 6).

\section{TDI mode characterization}

The TDI mode imaging is verified in laboratory by imaging a rotating cylinder with targets printed on its body. The rotation of the cylinder is in synchronization with the vertical charge transfer rate of the TDI detector. Figure 6 shows the image captured by OHRC.

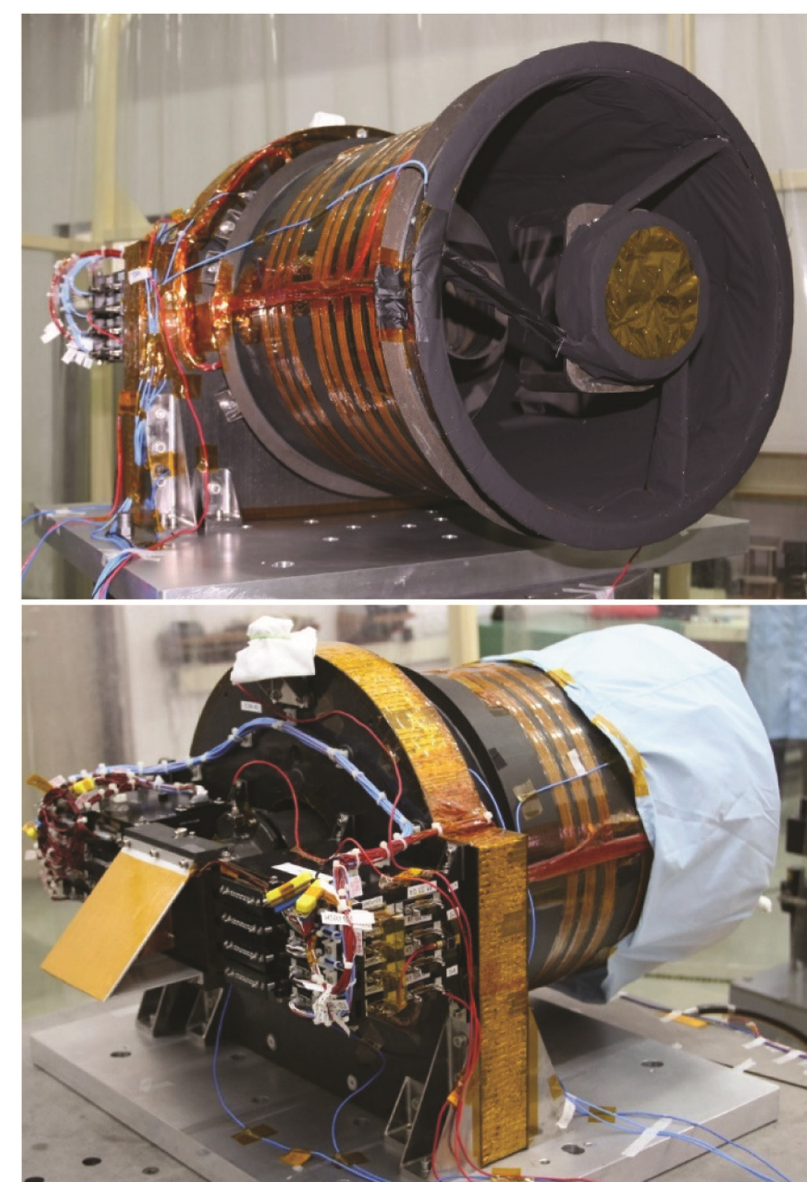

Figure 5. Realized OHRC payload.

Table 5. Camera mass, size and operating temperature

\begin{tabular}{lc}
\hline Parameter & Values \\
\hline Mass $(\mathrm{kg})$ & 17.5 \\
Size $\left(\mathrm{mm}^{3}\right)$ & $486 \times 463 \times 700$ \\
Operating temperature $\left({ }^{\circ} \mathrm{C}\right)$ & $10-30$ \\
\hline
\end{tabular}

\section{Spectral characterization}

Relative spectral response (RSR) is measured using a double monochromator-based spectro-radiometer system during spectral characterization activity. System level measured RSR is shown in Figure 7.

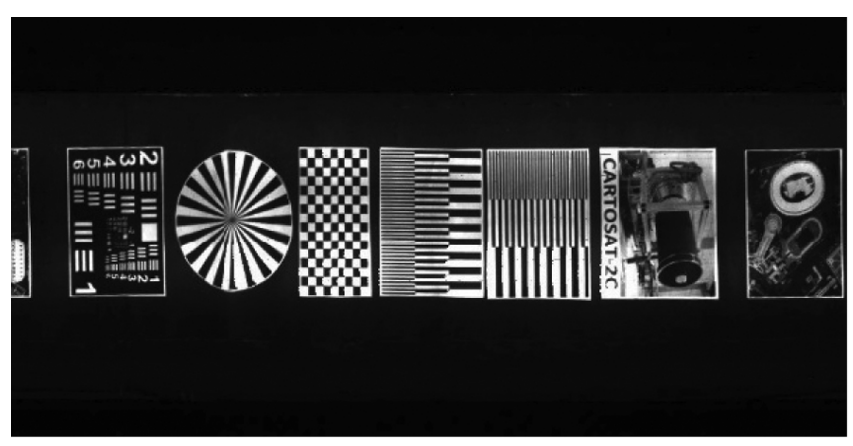

Figure 6. Image captured by OHRC in time delay integration mode of operation in laboratory.

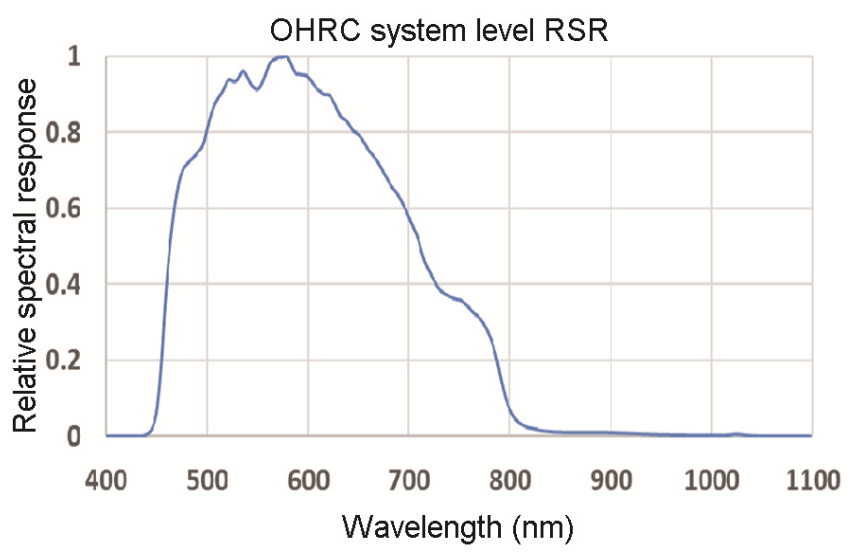

Figure 7. Relative spectral response of OHRC.

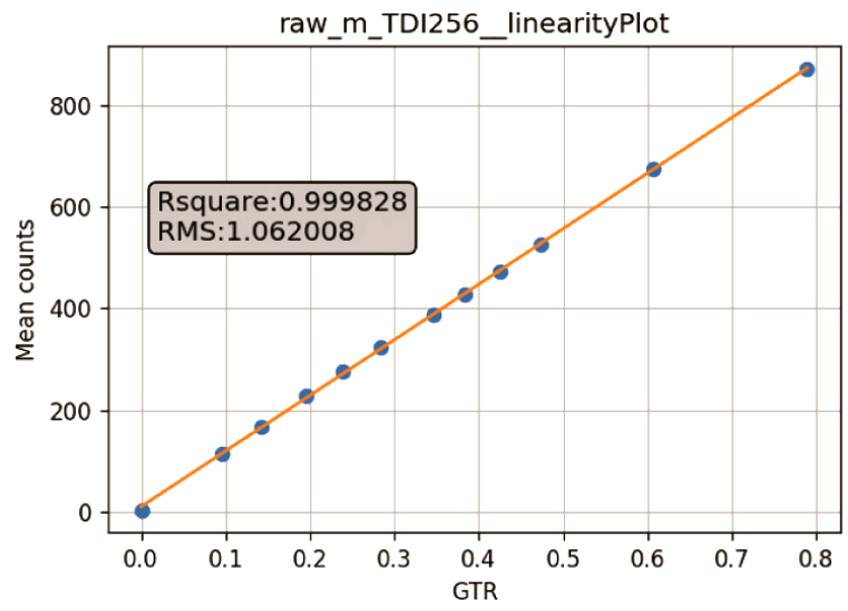

Figure 8. Linearity of the radiometric response of the OHRC (input radiance in $\mathrm{mW} / \mathrm{cm}^{2} / \mathrm{sr} / \mu \mathrm{m}$ on $x$-axis versus output counts (10 bit) on $y$-axis).

CURRENT SCIENCE, VOL. 118, NO. 4, 25 FEBRUARY 2020 
Table 6. Square wave response (SWR) of the OHRC

\begin{tabular}{lccc}
\hline & Extreme field (R1) & Centre field (R2) & Extreme field (R3) \\
\hline SWR (\%)@ Nq/2 & 28 & 34 & 30 \\
SWR (\%)@Nq & 13 & 16 & 14 \\
\hline
\end{tabular}

Table 7. Radiometric performance of OHRC, saturation radiance (SR) and SNR

\begin{tabular}{lc}
\hline Parameter & Values \\
\hline Reference radiance $\left(\mathrm{mW} / \mathrm{cm}^{2} / \mathrm{sr} / \mu \mathrm{m}\right)$ & $\sim 0.5$ \\
$\mathrm{SR}\left(\mathrm{mW} / \mathrm{cm}^{2} / \mathrm{sr} / \mu \mathrm{m}\right)$ & $0.8 @ 256$ TDI 3.2@64 TDI \\
$\mathrm{SNR}$ & $\sim 100$ (reference radiance) \\
& $(\sim 140$ at SR $)$ \\
\hline
\end{tabular}

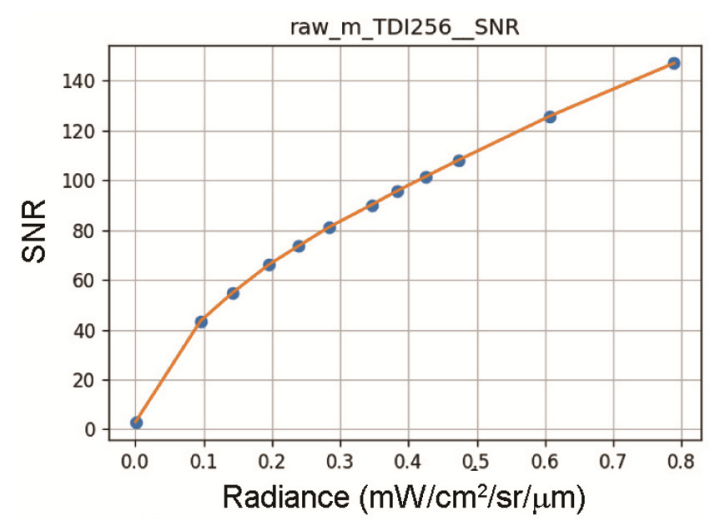

Figure 9. Radiometric performance in terms of the signal-to-noise ratio (on $y$-axis) at various input radiances in $\mathrm{mW} / \mathrm{cm}^{2} / \mathrm{sr} / \mu \mathrm{m}$ (on $x$-axis).

\section{Radiometric characterization}

Camera's radiometric response linearity (Figure 8), count (10 bit) to radiance coefficients and SNR (Figure 9) are measured during radiometric characterization activity using integrating sphere as uniform light source. This activity also known as light transfer characteristic and the performance is tabulated in Table 7.

\section{Data product}

Data processing for OHRC is planned for two different scenarios, one is for pre-landing decision support system (DSS) and other for regular operations. In DSS, two consecutive orbit datasets will be acquired and processed for the DEM and corresponding hazard map generation. A software pipeline has been developed to process OHRC DSS images $(\sim 3 \mathrm{~km} \times 12 \mathrm{~km})$, which is capable of generating DEM in $21 \mathrm{~min}$ after receiving the second orbit data. Regular operations products are according to defined levels. There are three levels $(0,1$ and 2$)$ defined for OHRC data products.

Level-0 dataset consists of payload raw data (as it is collected from payload), ancillary data (mainly SPICE kernels generated from the mission) and housekeeping data (health of the spacecraft). Level-1 data will be radiometrically corrected and geometrically tagged data. Radiometrically corrected datasets are free of photoresponse non-uniformity. Level-2 datasets include both radiometric and geometric correction. DEM generation and Ortho correction using Lunar control points or reference data are planned for stereo acquisitions.

\section{Summary}

OHRC flight model with $0.25 \mathrm{~m}$ GSD from $100 \mathrm{~km}$ is developed and characterized for optical, spectral and radiometric performance. This instrument provides mission-critical information with respect to hazard avoidance during landing, for which it has been tested and qualified through various tests.

ACKNOWLEDGEMENTS. We thank the contributions of all colleagues involved in the realization of the instrument. Several units of ISRO are involved in the development of the OHRC. The Sensors Development Area at Space Applications Centre is the lead area responsible for overall design, development, testing, qualification and delivery of the instrument. The optical elements are fabricated at LEOS, Bengaluru. The structure was developed at CMSE, Thiruvananthapuram. We gratefully acknowledge the support and guidance received from various areas and are thankful to the Director, Space Applications Centre for the constant encouragement.

Received and accepted 27 August 2019

doi: $10.18520 / \mathrm{cs} / \mathrm{v} 118 / \mathrm{i} 4 / 560-565$ 